\title{
Variations
}

Variations

Revue internationale de théorie critique

19 | 2016

Critique des humanités numériques

\section{Épistémologie du code et imaginaire des « SHS 2.0 »}

\section{Sébastien Broca}

\section{OpenEdition}

Journals

Édition électronique

URL : http://journals.openedition.org/variations/701

DOI : 10.4000/variations.701

ISSN : 1968-3960

Éditeur

Les amis de Variations

Référence électronique

Sébastien Broca, «Épistémologie du code et imaginaire des « SHS 2.0 » ", Variations [En ligne], 19 |

2016, mis en ligne le 06 avril 2016, consulté le 21 décembre 2020. URL : http://

journals.openedition.org/variations/701; DOI : https://doi.org/10.4000/variations.701

Ce document a été généré automatiquement le 21 décembre 2020.

Les ami•e•s de Variations 


\title{
Épistémologie du code et imaginaire des « SHS 2.0 »
}

\author{
Sébastien Broca
}

« Le succès de la physique mathématique a rendu

l'homme des sciences sociales jaloux de sa

puissance, sans qu'il comprenne vraiment les attitudes intellectuelles qui y avaient contribué.

L'usage de formules mathématiques avait accompagné le développement des sciences de la nature et devint la mode dans les sciences sociales,

exactement comme des peuplades primitives adoptent les modes occidentales d'habillement cosmopolite et le parlementarisme à partir d'un vague sentiment que ces accoutrements et rites magiques les porteront d'emblée au niveau de la

culture et de la technique modernes [...]»

Norbert Wiener

1 Faut-il avoir des compétences avancées en mécanique pour comprendre la place prise par la voiture dans nos systèmes de transport? Être capable d'opérer un patient pour réfléchir à l'organisation du système de santé ? Avoir un diplôme en agroforesterie pour s'alarmer des conséquences de la déforestation? De telles questions semblent passablement absurdes. Une injonction de ce type infuse pourtant un pan de la réflexion actuelle, notamment au sein du courant des humanités numériques (digital humanities - HN). Plusieurs auteurs soutiennent en effet que la connaissance et la pratique de l'informatique sont devenus des prérequis afin de comprendre - et éventuellement de critiquer - le monde social dans lequel nous vivons, dans la mesure où celui-ci est dorénavant marqué par l'omniprésence des logiciels (Fuller, 2008; Andreessen, 2011). Le théoricien des médias Friedrich Kittler affirme ainsi que ce n'est qu'à condition de connaitre au moins deux langages de programmation que les étudiants peuvent être "capables de dire quelque chose sur ce qu'est la "culture" actuellement» (Kittler, in Manovich, $2013: 20$ ). Le spécialiste des digital humanities Stephen Ramsay (2011) présente 
le fait de savoir coder comme une condition sine qua non pour pouvoir se dire « humaniste numérique ». Un peu moins péremptoire, le chercheur David M. Berry se demande si nous ne sommes pas « en train de voir émerger le fait de lire et d'écrire du code de logiciel comme faisant partie de la pédagogie requise pour générer une nouvelle subjectivité computationnelle critique et réflexive au sein de l'université » $(2015: 199)$.

Cete injonction à "faire du code" n'est pas sans lien avec le projet général des humanités numériques. Pour ce courant de recherche, la « logiciarisation » du monde est souvent à la fois un objet et une méthode. Les digital humanities se proposent en effet d'étudier notre société en tant qu'elle "peut être caractérisée comme une société du logiciel (software society)", et notre culture en tant qu'elle "peut légitimement être appelée une culture du logiciel (software culture)» (Manovich, 2013 : 33). En d'autres termes, il s'agit d'appréhender l'humanité «sous les conditions ouvertes par la numérisation du signe, la calculabilité et les formes sémiotiques qui leurs sont liées » (Granjon, dans ce numéro). Les méthodes employées pour conduire ce projet laissent la part belle aux technologies informatiques. Il s'agit de renouveler les approches classiques en humanités et en sciences sociales grâce aux nouveaux outils numériques mis à la disposition des chercheurs: analyse de contenus grâce au text mining, exploitation de bases de données grâce au data mining, outils de topic modelling (modélisation des thèmes) en littérature, représentations visuelles ou cartographiques de certains phénomènes sociaux, etc. En bref, la logiciarisation du monde va ici de pair avec une logiciarisation de l'appréhension savante du monde.

3 Les enjeux de cet usage d'outils informatiques au sein des sciences humaines sont parfois abordés avec quelque légèreté. Les auteurs $d u$ «manifeste pour des humanités numériques 2.0 " proposent ainsi d'exploiter «les boîtes à outils numériques pour les mettre au service des spécificités méthodologiques qui font le propre des humanités » (Schnapp et al., 2008). Une telle formulation fait comme si les technologies informatiques étaient sans influence sur l'orientation épistémologique générale des recherches qu'elles outillent. Or rien n'est moins vrai. Les outils numériques ne sont pas « neutres ». Ils sont solidaires de pratiques spécifiques et emportent avec eux des manières de se rapporter au monde et de concevoir la connaissance. Ils ne sauraient donc être sans influence sur l'épistémologie des digital humanities. De nombreux auteurs font, du reste, clairement apparaître cet enjeu. Tarleton Gillespie remarque que les «algortihmes ne nous aident pas seulement à trouver de l'information " mais "fournissent aussi des moyens pour savoir ce qu'il y a à savoir et comment le savoir» (2014: 167). Lev Manovich relève que l'appareillage technologique contemporain introduit « de nouvelles manières d'acquérir du savoir, et dans ce processus redéfinit ce qu'est le savoir » $(2013: 338)$. Katherine Hayles insiste sur le fait que « les ordinateurs ne sont plus de simples outils (s'ils l'ont jamais été) mais des systèmes complexes qui produisent de plus en plus les conditions, idéologies, affirmations et pratiques qui aident à constituer ce que nous appelons la réalité » (2005: 60). En bref, il est assez évident qu'on ne peut pas dissocier les choix épistémologiques des moyens grâce auxquels la connaissance est produite (Waquet, 2015), et il semble particulièrement judicieux de rappeler cette vérité générale dans le cadre des humanités numériques (Granjon, 2015).

4 On peut en effet tracer un parallèle entre l'informatique et certaines recherches menées à propos de l'écriture. L'anthropologue Jack Goody (1978) a montré que l'invention de celleci s'est accompagnée de l'émergence d'un type particulier de rationalité, que l'on nomme en français la «raison graphique». Autrement dit, l'écriture est allé de pair avec le 
développement d'un certain rapport au monde et avec l'apparition de structures conceptuelles spécifiques. Il est aujourd'hui envisageable d'adresser aux technologies informatiques une question similaire à celle abordée par Goody, en se demandant « en quoi le recours à des représentations calculées» s'accompagne également d'« une rationalité particulière » (Bachimont, 2004). Plusieurs notions ont ainsi été proposées ces dernières années pour faire sens de ce que l'utilisation de l'informatique «fait » à notre rapport au savoir et à la pensée. On a pu parler de "raison computationnelle» (Bachimont, 2004), de «pensée computationnelle» (Wing, 2006) ou encore de « rationalité algorithmique» (Rouvroy, 2011). Toutes ces expressions dirigent notre attention vers une même question, que j'aborderai pour ma part en parlant d'« épistémologie du code ». À travers cette expression, j'entends signifier que l'usage des technologies informatiques est bien solidaire d'une certaine conception du savoir (fut-elle implicite et non reconnue comme telle), et essayer de préciser les principales caractéristiques de celle-ci.

5 Avant d'aller plus loin dans cet effort de caractérisation, il est nécessaire de dire quelques mots de la manière dont l'épistémologie du code questionne les fondements traditionnels des sciences humaines. Comme l'ont souligné nombre d'analystes des HN, l'informatique semble introduire dans le champ des "sciences molles " le rapport au monde et à la connaissance propre aux «sciences dures » (Guichard, 2014), en rompant la séparation entre «deux cultures » que l'on a pour habitude de considérer assez étanches l'une à l'autre (Snow, 1959). Lorsqu'on confronte certaines caractéristiques relativement évidentes de la pratique informatique et des humanités, il est vrai que de nombreux antagonismes se font jour: les nombres s'opposent aux mots, le calcul contraste avec l'argumentation, le hack se différencie du yack, l'univocité du code tranche avec l'équivocité du langage courant, l'impartialité des données remet en cause l'engagement de l'intellectuel, la production collaborative de logiciels remplace le labeur solitaire de l'auteur... Tout paraît donc indiquer une tension épistémologique importante, et notre propos est d'y regarder de plus près, en examinant ce qu'implique l'usage de technologies informatiques au sein des sciences humaines, particulièrement dans le champ labellisé «humanités numériques». Je tenterai d'éclairer cette question en m'appuyant sur la philosophie de Cornelius Castoriadis et sur la distinction qu'elle opère entre deux dimensions de l'institution sociale: la dimension «ensembliste-identitaire» et la dimension «imaginaire » (1975). À travers ces termes au sens peu évident, Castoriadis théorise le mode d'être propre au monde social-historique (l'« imaginaire ») et montre que celui-ci ne peut jamais être complètement et adéquatement saisi par les opérations de la logique mathématique (l'« ensembliste-identitaire ») et, au-delà, par le recours à la formalisation, à la quantification et au calcul.

6 L'apport de Castoriadis pour le sujet qui nous occupe va plus loin. En montrant l'imbrication des dimensions imaginaire et ensembliste-identitaire, il nous permet également de penser que l'informatique, symbole de la rationalité froide et neutre de la machine, ne peut être découplée des significations qui sont projetées sur son usage. En d'autres termes, il faut considérer la rationalité de l'informatique mais aussi l'imaginaire de l'informatique. Une réflexion sur l'épistémologie du code doit par conséquent embrasser deux choses: les caractéristiques des opérations de connaissance permises par les ordinateurs; le sens dont ces opérations et leurs résultats sont investis au sein de nos sociétés, notamment par les praticiens de l'informatique. On voit par là que l'interrogation épistémologique ne peut se passer, ni d'une armature philosophique 
robuste, ni d'un tissu sociologique finement maillé. C'est certainement beaucoup demander. Je n'escompte donc que fournir quelques éléments à même de nourrir la réflexion sur un sujet qui mérite sans doute que l'on s'y arrête.

\section{La spécificité du monde social-historique}

\section{Les deux dimensions de l'institution sociale}

7 L'institution de la société se déploie selon Cornelius Castoriadis à la fois dans la dimension « ensembliste-identitaire» et dans la dimension proprement "imaginaire». Il faut entendre par là que deux logiques sont à l'œuvre dans toute société. «Brièvement parlant : il n'y a pas de société sans arithmétique ; et il n'y a pas de société sans mythes » (Castoriadis, 2005: 72). Castoriadis décrit la logique «ensembliste-identitaire» en référence à la théorie mathématique des ensembles, dont la première définition par Cantor était la suivante : « une collection en un tout d'objets définis et distincts de notre intuition ou de notre pensée » $(1895: 481)$. Il ne faut toutefois pas se laisser abuser par cette référence. La dimension « ensembliste-identitaire » n'a évidemment rien à voir avec la personne de Cantor en tant que telle. Il se trouve simplement que celui-ci a formalisé en mathématiques, de manière aussi « pure » que possible, la logique qui caractérise selon Castoriadis une « dimension essentielle et inéliminable non seulement du langage, mais de toute vie et de toute activité sociale» (1975 : 327-328). Cette dimension tient au fait que tout groupement humain doit être en mesure de considérer des choses bien déterminées, de les ranger par classes, de leur attribuer certaines propriétés, et de considérer certaines relations entre ces éléments comme stables. En d'autres termes, en tant qu'elle est traversée par la logique ensembliste-identitaire, une société fonctionne sous le règne de la déterminité (toute chose " $\mathrm{X}$ » est déterminée par un ensemble de propriétés qui la distinguent de " $\mathrm{y} \|)$ et en vertu des règles élémentaires de la logique (principes du tiers exclu, d'appartenance, d'inclusion, syllogistique, etc.).

Cette dimension est présente, bien qu'à des degrés divers, dans l'organisation de toutes les sociétés, sans quoi celles-ci seraient pur chaos. Pour qu'une société s'institue, il faut qu'elle puisse dire les objets, les compter, les rassembler, les distinguer. Ainsi, même dans les groupements humains qui apparaissent à la pensée occidentale moderne comme les moins rationnels, une vache fait partie de l'ensemble "vache ", elle se distingue par là des éléments réunis dans l'ensemble "taureau», donne sans l'ombre d'un doute naissance à des veaux et des génisses, non à des grenouilles, etc. De même, l'eau coule, le feu brûle, «l'ensemble des cases forme le village qui est ce village et notre village, celui auquel nous appartenons et auquel n'appartiennent pas ceux de l'autre village [...]» (Castoriadis, 1975: 336). L'institution sociale présuppose ainsi toujours «des objets distincts et définis, pouvant être collectés et former des touts, composables et décomposables, définissables par des propriétés déterminées et servant de support à la définition de celles-ci » (Castoriadis, 1975: 336). Cette logique ensembliste-identitaire n'opère que parce qu'elle se rapporte à un monde qui est déjà « ensemblisable », parce que ce dernier se prête en quelque sorte à ses opérations. Cette forme d'ajustement ou de correspondance entre la dimension ensembliste-identitaire et le monde est ce que Castoriadis appelle « l'étayage de la société sur la première strate, ou strate naturelle, du donné » (Castoriadis, 1975 : 335). La présence de l'ensembliste-identitaire dans toutes les sociétés nous révèle ainsi que le monde ne peut être organisé que parce qu'il est 
organisable, parce qu'il y existe des éléments distincts possédant des propriétés stables : "l'entendement serait sans objet, si le monde était pure multiplicité du divers, de l'absolument divers » souligne Castoriadis (1997: 13). Cela nous montre aussi qu'il existe des limites «naturelles» à ce que peut l'institution sociale: aucune société ne peut décider qu'il n'y aura plus qu'un seul sexe ou que le soleil ne se lèvera pas le jour suivant la mort du roi...

9 Si la logique ensembliste-identitaire saisit une dimension importante de l'institution sociale et de la manière dont celle-ci prend appui sur le monde, elle ne suffit pas à élucider ce par quoi une société se fait elle-même comme un tout cohérent, semblable à aucun autre. L'institution sociale se déploie en effet dans une deuxième dimension, qui est la dimension proprement imaginaire. Là-encore, il ne faut pas se laisser abuser par la terminologie de Castoriadis. Le terme "imaginaire» ne désigne pas quelque chose de fictif, d'irréel ou de spéculaire, mais le mode d'être des significations qui font tenir ensemble toute forme sociale-historique. En effet, ce qui fait le propre d'une société et lui donne une cohérence, ce sont les "significations imaginaires sociales» (S.I.S.) qui l'animent. Il faut entendre par là qu'il existe un «tissu de sens, ou de significations, qui pénètrent toute la vie de la société, la dirigent et l'orientent " (Castoriadis, 2005 : 67). Les S.I.S. représentent ainsi la manière, historiquement spécifique, dont une société donne sens aux choses et répond aux questions essentielles qu'elle doit affronter : son identité, son rapport au monde, ses normes, ses valeurs, ses besoins, ses désirs... Ces significations sont « imaginaires » en un sens très précis. Il faut comprendre par là qu'elles sont de l'ordre d'une création non déterminée. Autrement dit, elles ne peuvent se dériver simplement, ni d'un certain état de la réalité, ni d'une considération de la rationalité, ni d'une exigence fonctionnelle. Pour Castoriadis, "imaginaire» signifie "position de nouvelles formes, et position non déterminée mais déterminante; position immotivée, dont ne peut pas rendre compte une explication causale, fonctionnelle ou même rationnelle» (Castoriadis, 1996 : 159). Les S.I.S. ne sont donc pas la conséquence d'une « nature humaine » ou d'un « ordre naturel », pas plus qu'elles ne dérivent d'une simple exigence de conservation de la société, ou d'une considération anhistorique de la rationalité. Elles ne sont pour ainsi dire la conséquence de rien (bien que l'on puisse évidemment s'efforcer de retracer l'évolution historique des S.I.S. dans une société donnée). Elles sont une création de ce que Castoriadis appelle "l'imaginaire social instituant ", c'est-à-dire le collectif anonyme et impersonnel que constitue une société. Leur source est tout simplement l'activité créatrice de sens des hommes. Elles témoignent $\mathrm{du}$ fait que l'histoire est création, surgissement du nouveau, qu'elle est la manière dont l'humanité se transforme sans que jamais une forme sociale-historique donnée n'apparaisse comme la simple déduction logique de celle qui la précède. Toute société se fait grâce à la position de significations imaginaires et existe ainsi par-delà la déterminité, c'est-à-dire par delà la dimension ensembliste-identitaire.

Les S.I.S. constituent en effet une diversité pour partie non ensemblisable. Elles ne sont pas bien distinctes et déterminées, mais au contraire « indéfiniment reliées les unes aux autres moyennant un mode de relation qui est le renvoi » (Castoriadis, 2005 : 73). Ainsi, " la signification "prêtre" me renvoie à la signification "religion", qui me renvoie à Dieu, qui me renvoie à je ne sais pas quoi, mais certainement aussi en tout cas au monde en tant que sa création - donc aussi par exemple au pêché » (Castoriadis, 2005: 73). Les S.I.S forment donc un type particulier d'organisation que Castoriadis appelle le magma, soit une organisation « qui n'est pas réductible à des ensembles ou des systèmes d'ensembles, 
aussi riches et complexes soient-ils» (Castoriadis, $1986: 231$ ). Autrement dit, un magma n'est pas une collection close d'éléments bien déterminés, exhaustivement dénombrables, liés les uns aux autres par des relations nécessaires. Il fonctionne par une multiplicité de renvois entre des significations partiellement enchevêtrées. Les magmas de S.I.S. ne sont par conséquent «pas reconstructibles par des opérations logiques» (Castoriadis, 2005 : 73)

\section{La double nature du langage}

11 Un exemple particulièrement éclairant pour saisir la différence et la coexistence entre la dimension ensembliste-identitaire et la dimension imaginaire est celui du langage. Celuici relève en effet simultanément des deux logiques, ce que Castoriadis exprime en disant qu'il est à la fois code et langue. Il "est code en tant qu'il organise et s'organise identitairement, c'est-à-dire en tant qu'il est système d'ensembles (ou de relations ensemblisables) » (Castoriadis, $1975:$ 352). Sous cet aspect, le langage se présente comme une collection d'éléments, sonores et significatifs, qui peuvent être isolés. Il est une composition de ces unités distinctives (phonèmes, morphèmes, mots), entre lesquelles il établit des relations définies. Le langage fonctionne ainsi comme un code aux niveaux morphologique, phonétique et syntaxique (Tomès, $2007: 133$ ). Il opère également, pour partie, comme un code du point de vue de la signification. En effet, une signification ne peut entrer dans le langage que si, « par un de ses aspects, [...] elle se laisse saisir comme si elle était quelque chose de distinct et défini, sans quoi on ne saurait plus de quoi on parle » (Castoriadis, $1975:$ 359). Autrement dit, le langage comprend et « institue toujours une dimension d'univocité » (Castoriadis, 1975 : 352). Il faut noter que cette dimension ensembliste-identitaire du langage n'est pas totalement dénuée de puissance générative. Comme le souligne Castoriadis, un code peut intégrer "des opérations déterminées d'engendrement de nouveaux termes à partir d'une 'base' (famille d'éléments) donnée et fixe » (Castoriadis, 1975 : 322). Le langage se présente donc aussi, en tant que code, comme une série d'opérations réglées sur des éléments définis. Ces opérations peuvent produire une certaine forme de nouveauté, non pas absolue, mais déterminée par les propriétés des éléments combinés et les règles présidant à leur combinaison.

Cette nouveauté relative est toutefois loin de rendre compte de la richesse et de la créativité du langage. Celui-ci est aussi langue, ce par quoi il est irréductible tant «à une totalité fermée de significations fixes, déterminées, disponibles » (Castoriadis, 1975 : 321) qu'à une vaste combinatoire à partir de ces éléments préexistants (Castoriadis, 1975 : 323). La langue, c'est pour Castoriadis la composante dynamique et (véritablement) créative du langage, ce par quoi celui-ci se transforme; ce qui fait que «le lexique des significations est partout ouvert " (Castoriadis, $1975: 360$ ). En un mot, c'est sa dimension imaginaire. Lorsqu'on aborde cette composante, on quitte le sol de la déterminité au profit de la logique des magmas. Castoriadis souligne ainsi que la signification d'un mot n'est jamais totalement fixe, qu'on ne peut la dériver simplement d'un état préexistant de la langue, ni lui assigner de véritable limite, puisque cette signification renvoie à une multiplicité d'autres significations, qui renvoient elles-mêmes à d'autres significations, etc. Il faut par conséquent reconnaître que le langage humain ne se réduit pas à un code, à un ensemble d'éléments univoques susceptibles d'être combinés grâce à un nombre fini de règles. L'inventivité sémantique, l'ambiguïté et les glissements de sens sont des caractéristiques indépassables du langage, et ces caractéristiques renvoient au mode 
d'être spécifique du monde social-historique dans ce qui le différencie de la strate naturelle. Le langage nous dit que chaque société institue un magma de significations, dont la richesse ne saurait être épuisée - ni même valablement approchée - par la logique ensembliste-identitaire :

«Il n'y a langue qu'en tant qu'elle s'oppose au code, que pour autant qu'il y a autre chose que l'univoque. Ce n'est pas seulement que l' 'équivoque', l' 'ambiguïté', bref la polysémie au sens large en sont inéliminables; elles ne sont pas des défauts ou des scories, elles sont positivement constitutives de la langue comme langue. En elles s'instrumente, s'incarne et, j'ose dire, s'éclaire en partie le mystère de la double fonction de la langue, comme milieu ou élément à la fois de l'imaginaire et du rationnel, de la poésie et de la vérité, comme aussi - ce n'est pas la même articulation - du sujet et du réel. Car la polysémie n'est pas seulement le sang de la poésie, mais aussi ce qui rend possible la présence dans le langage de significations vraies, c'est-à-dire non algorithmiques, c'est-à-dire qui renvoient toujours à autre chose, à partir de quelque chose » (Castoriadis, 2007 : 158).

Il faut donc reconnaitre la double nature du langage et, à travers lui, de la signification. Celle-ci ne peut se dire qu'en prenant appui sur la logique ensembliste-identitaire (sans quoi l'on ne pourrait jamais savoir de quoi l'on parle), mais est toujours en excès sur cette logique, dans la mesure où elle n'est pas parfaitement distincte, circonscrite et close sur elle-même.

\section{L'imaginaire et le travail des sciences sociales}

14 L'exemple du langage signale que, s'il est crucial de distinguer dimension ensemblisteidentitaire et dimension imaginaire, il importe tout autant de saisir leur profonde intrication. Castoriadis souligne, en usant d'une métaphore topologique, que ces dimensions sont « partout denses » dans la vie sociale (1986:230). Autrement dit, il y a de la logique ensembliste-identitaire dans les significations imaginaires - «il n'y a pas de mythe sans arithmétique; tout mythe est obligé de faire appel aux mêmes schèmes qui sont à la base de l'arithmétique, et même, explicitement, aux nombres " (Castoriadis, 2005 : 72) - et, réciproquement, il y a de l'imaginaire dans les activités sociales qui nous semblent les plus «logiques» ou les plus «rationnelles». Ainsi, les expressions privilégiées de la logique ensembliste-identitaire, comme les mathématiques ou l'informatique, «baignent ", comme l'écrit parfois Castoriadis, dans le magma des S.I.S. Ces significations déterminent la place qui est socialement dévolue à ces activités, voire altèrent leur contenu même. Pour le comprendre, considérons les sociétés occidentales capitalistes modernes telles qu'elles se sont déployées depuis la Renaissance. L'une des S.I.S. centrales de ces sociétés est selon Castoriadis « l'expansion illimitée de la maitrise rationnelle» (1990: 19); signification que l'on peut approcher à travers la fameuse formule de Descartes: "se rendre comme maitres et possesseurs de la nature ». Ce schème imaginaire a un lien très étroit avec nombre de phénomènes caractéristiques de la modernité capitaliste: le développement sans limite de la production et la consommation par exemple, mais aussi l'essor fabuleux des sciences et des techniques. Autant qu'on puisse en juger, cet essor ne se serait en effet jamais produit si les sociétés n'avaient investi de sens et de valeur la compréhension et la domestication de la nature, selon des modalités relevant - pour le dire rapidement - de la logique mathématique et de la rationalité instrumentale. Autrement dit, «derrière " la science, il y a toujours de l'imaginaire, y compris s'agissant des formes de connaissance qui apparaissent comme emblématiques de la logique ensembliste-identitaire (mathématiques, physique, 
informatique, etc.). Il existe, pour le dire d'un trait, une dépendance de la science à l'égard « des lignes de force idéologiques-imaginaires du champ historique » (Castoriadis, 1998: 284). L'essor fantastique de la formalisation, de la quantification et de la discrétisation - manifestations idéales-typiques de la logique ensembliste-identitaire - ne peuvent ainsi se comprendre indépendamment de l'idée que tout serait calculable, idée qui ne peut être qu'une signification imaginaire sociale, puisqu'on ne peut la dériver d'une considération de l'en-soi des choses. S'il y a donc bien de la logique ensemblisteidentitaire lovée dans l'imaginaire, il y a aussi de l'imaginaire à l'œuvre dans le déploiement de la logique ensembliste-identitaire, notamment dans les sciences dites « exactes ».

L'ensemble de ces considérations permet de mieux aborder l'objet des sciences sociales ainsi que leur spécificité épistémologique. Si l'on accepte l'ontologie du social-historique proposée par Castoriadis, ce sont les significations imaginaires qui apparaissent comme l'objet privilégié de ces disciplines. Comprendre les spécificités des diverses sociétés dans l'histoire revient à élucider les schèmes imaginaires par lesquels celles-ci donnent sens aux choses, et à exposer la manière dont ces schèmes irriguent et orientent le fonctionnement concret des institutions politiques, religieuses, scientifiques, etc. Ce projet ne peut être mené que moyennant une épistémologie propre, adéquate au mode d'être des S.I.S. Comme le souligne Castoriadis, « réfléchir vraiment la société et l'histoire, c'est essayer de réfléchir le social-historique dans une dimensionnalité dont nulle par ailleurs nous ne trouvons d'exemple " (Castoriadis, 1998: 262). On comprend, dès lors, pourquoi les sciences sociales sont - ou devraient être - rétives à certains types de formalisation et de quantification. Celles-ci «laissent hors d'atteinte l'essentiel» (Castoriadis, 1998 : 265), à savoir la logique propre au monde social-historique. Penser celui-ci implique de dépasser la logique ensembliste-identitaire, de rompre avec une définition de la pensée comme calcul, et de ne pas se laisser impressionner par l'illusion d'un usage univoque et parfaitement systématique du langage (Tomès, 2007 : 139-140). Cela signifie plus concrètement que l'on ne peut simplement transposer dans le domaine des sciences humaines des formalisations qui semblent avoir réussi dans d'autres champs scientifiques, ou qu'il faut, à tout le moins, s'interroger sur la validité de telles transpositions et sur ce qu'elles laissent de côté.

\section{L'épistémologie du code}

\section{La rationalité algorithmique}

Cet excursus dans la philosophie de Cornelius Castoriadis fournit quelques bases solides pour caractériser l'épistémologie du code et en dégager les enjeux dans le champ des humanités. Il apparaît ainsi que l'informatique peut être abordée comme une mise en œuvre, fantastiquement efficace, de la logique ensembliste-identitaire. Les ordinateurs sont en effet des "machines algorithmiques », c'est-à-dire des technologies permettant de «lire et stocker des données, leur appliquer des procédures mathématiques de manière contrôlée, et offrir de nouvelles informations à la sortie » (Gillespie, 2014). La puissance de ces procédures pour résoudre un grand nombre de problèmes n'est plus à démontrer. Elle a pour condition le fait de fournir à la machine des données qu'elle soit en mesure de lire et de traiter, à savoir des éléments distincts et définis à partir desquels des calculs sont possibles. 
17 Cela passe par un travail qu'Antoinette Rouvroy décrit comme une "opération de purification » : un "travail de suppression de toute signification, afin que ces données brutes puissent être calculables et fonctionnent non plus comme des signes qui signifient quelque chose en rapport à ce qu'ils représentent, mais comme quelque chose qui se substitue à la réalité signifiante, la fasse disparaître" (Rouvroy, Stiegler, 2015). Autrement dit, l'informatique repose techniquement et logiquement sur la distinction entre la forme d'une donnée et sa signification, et sur la possibilité de mettre cette dernière entre parenthèses. L'ordinateur requiert de réduire la langue au code, de manipuler des symboles « comme s'ils étaient algébriques [...] alors qu'ils ne le sont pas» (Bachimont, 2004). La disparition de la signification n'est toutefois que provisoire. Lorsqu'il considère les outputs de ces procédures de calcul, le sujet humain disposent à nouveau d'une capacité à les faire signifier d'une multitude de manières, c'est-à-dire à les interpréter en vertu de règles non algorithmiques. De plus un code peut, comme noté supra, engendrer de nouveaux termes à partir d'un ensemble fini d'éléments déterminés. La rationalité algorithmique de l'ordinateur est donc susceptible de produire des données inédites, dont la nouveauté relative - car déterminée par les éléments combinés et les règles de calcul suivies - servira parfois d'appui pour la création de nouvelles significations. Toutefois, la formalisation et la procéduralisation qui sont au cœur même de l'informatique réduisent aussi l'éventail des significations. Ce qui a été perdu dans l'opération de purification menée en amont, afin de rendre les données "computables", ne se retrouve pas forcément en aval. Une fois qu'elles sont passées sous les fourches caudines du traitement algorithmique, les données ne peuvent pas toujours être à nouveau reliées à leur contexte originel, aux vies singulières dont elles étaient la traduction et à la richesse de sens qui était la leur. L'informatique n'implique donc pas qu'une éclipse de la signification, elle en est parfois (bien que pas toujours) un appauvrissement durable. Dès lors qu' " au niveau du code binaire, le système ne peut tolérer que peu, sinon aucune ambiguïté » (Hayles, 2005 : 46), rien ne garantit que la richesse de la langue, au sens de Castoriadis, se retrouve ex post. Aussi, si Alexander Galloway a pu écrire très justement que «le code est le seul langage qui est exécutable» (Galloway, $2004: 165)$, il faut y ajouter : parce qu'il est le seul langage dont la dimension de la langue est absente.

\section{L'imaginaire de l'informatique}

À un premier niveau, celui de la machine, l'épistémologie du code se caractérise donc par une rationalité algorithmique, donc l'exercice suppose la mise entre parenthèses de la signification. On ne peut cependant en rester là. L'épistémologie du code, ce sont aussi les attitudes face à l'informatique et au savoir de ceux qui écrivent et utilisent des logiciels. Ce deuxième aspect renvoie à ce que nous appelons, après d'autres, l'imaginaire de l'informatique. Philippe Breton a décrit cet imaginaire comme organisé autour de trois idées : la "vision d'une société future radicalement transformée » par l'informatique, la «comparaison ente le cerveau et l'ordinateur", la conviction que «la logique est une valeur universelle, un outil de compréhension du monde autant qu'un moyen de le transformer » (Breton, 1990 : 157).

19 Le premier trait fait directement écho à ce qu'affirment aujourd'hui de nombreux promoteurs des software studies et des digital humanities, pour qui la place prise par les logiciels dans nos vies constitue la principale caractéristique et la grande originalité des sociétés actuelles (Manovich, 2013). Si ce «constat» sur la logiciarisation du monde ne 
peut être totalement détaché de la volonté d'asseoir un nouveau champ de recherche (le créneau des $\mathrm{x}, \mathrm{y}$ ou $\mathrm{z}$ studies est, on le sait, très encombré), il montre aussi que ceux qui ont une grande familiarité avec l'informatique sont bien souvent les premiers à y voir une force majeure de transformation sociale. Ainsi, le fait de prendre comme objet de recherche la "software society " participe déjà dans une certaine mesure de l'imaginaire de l'informatique. Le deuxième trait - la comparaison entre le cerveau et l'ordinateur évoque quant à lui les grandes heures des programmes de recherche en intelligence artificielle, notamment ceux dits « computationnalistes» (Varela, 1988: 36). Il renvoie également à une idée bien plus ancienne : celle selon laquelle la pensée serait réductible à un calcul, comme le soutient par exemple Hobbes au début du Léviathan (Hobbes, 2000 : 110-111). On voit par là que loin d'être une simple conséquence de l'invention de l'ordinateur, l'imaginaire de l'informatique plonge ses racines dans certaines caractéristiques profondes de la modernité occidentale. C'est le troisième trait - la croyance dans l'universalité de la logique - qui me semble toutefois le plus intéressant. Breton précise son contenu en rappelant que les informaticiens et certains dirigeants étaient durant les années 1960 convaincus que tout était calculable, que le «calcul logique » était «l'une des clefs de compréhension de l'univers tout entier» (1990:225). Cette posture épistémologique faisait écho à celle des pères spirituels de l'ordinateur, Alan Turing et John von Neumann, qui accréditèrent en leur temps l'idée selon laquelle les nouvelles machines allaient « confirmer la justesse et l'universalité de la démarche scientifique tout entière ", en permettant à la science de "prendre pour objet [...] des domaines qui lui avaient toujours échappé : l'homme, la société, la connaissance ellemême » (Breton, $1990: 164$ ).

Il n'est pas certain que les choses aient tant changé aujourd'hui. Dans un ouvrage imposant paru en 2002, le spécialiste des automates cellulaires Stephen Wolfram présente ainsi ce qu'il nomme le "principe d'équivalence computationnelle ». La formulation la plus simple de ce principe est la suivante : «tous les processus, qu'ils soient le fruit des efforts humains ou qu'ils se déroulent spontanément dans la nature, peuvent être appréhendés comme des processus computationnels » (2002 : 715). L'idée selon laquelle le monde naturel et le monde humain seraient pareillement «computables» semble donc avoir encore quelques beaux jours devant elle. Bien entendu, tous les programmeurs n'adhèrent pas à cette vision maximaliste. Il n'empêche qu'ils ont souvent une disposition d'esprit qui les pousse à aborder les questions extérieures au champ informatique, en usant des catégories propres à leur discipline et en suivant certains réquisits de la rationalité algorithmique. L'anthropologue Gabriella Coleman (2004) le fait bien comprendre dans l'un de ses nombreux articles consacrés aux hackers. Elle y explique l' «agnosticisme politique» de nombre d'entre-eux par les ambiguïtés, blocages et incohérences propres à l'action politique, alors que « la programmation est considérée comme un domaine de pensée et d'expression transparent, neutre, et maîtrisable». Lorsqu'ils se lancent malgré tout dans le champ politique, les hackers tentent ainsi d'y importer certains principes et outils éprouvés dans l'écriture du code, comme l'ont revendiqué les activistes de l'association La Quadrature du Net en France: "Par notre culture du logiciel libre, et par nos outils qui sont ceux du logiciel libre (canaux IRC, Wikis, Svn, Drupal), nous construisons notre action politique et menons cette mission de production de savoirs sur le processus législatif, sur le processus politique, et de savoirs citoyens sur comment agir en tant qu'individu " (Zimmermann, 2010). Richard Stallman, le créateur du mouvement du logiciel libre, révèle d'une autre manière la tournure d'esprit associée à la pratique de l'informatique, par son souci quasi obsessionnel de la 
précision terminologique. Il tient ainsi une liste des «mots à éviter » (Words to Avoid) en raison de leur " ambiguïté »" On peut voir là une tentative mi-sérieuse, mi-fantasque, de faire fonctionner la langue avec la clarté, l'univocité et la transparence du code ; tentative qui, si elle renvoie avant tout au personnage singulier qu'est Stallman, ne semble pas non plus tout à fait sans lien avec l'imaginaire plus large de l'informatique, dans sa tendance à vouloir organiser le monde en une collection d'éléments bien distincts et déterminés.

\section{Les Sciences humaines et sociales 2.0}

\section{Un savoir construit sur le modèle du logiciel (libre)}

L'épistémologie du code combine donc la rationalité algorithmique de la machine avec l' imaginaire de l'informatique tel qu'il s'est déployé depuis le milieu du $\mathrm{XX}^{\mathrm{e}}$ siècle. Reste à examiner dans quelle mesure cette épistémologie imprègne le courant des humanités numériques. Certains textes laissent en la matière peu de place au doute. Le «manifeste pour des humanités numériques 2.0 » moque ainsi les «savants uni-medium (probablement l'imprimé) », "abrutis par des siècles de somnolence », qui « n'ont que très peu, voire jamais, mis au point de logiciels, analysé du codage, créé une base de données ou conçu une interface utilisateur " (Schnapp et al., 2008). Passons rapidement sur l'idée selon laquelle avant l'invention de l'informatique, c'est bien connu, les philosophes, historiens et autre sociologues faisaient tranquillement la sieste dans des bibliothèques poussiéreuses... Même en reconnaissant certaines vertus à l'outrance, on peine à ne pas trouver ridicule cette disqualification sommaire de tout chercheur ne disposant pas de compétences en programmation. D'autres passages du même " manifeste » sont néanmoins plus intéressants, en ce qu'ils permettent d'aborder avec davantage de finesse le rapport des HN à l'épistémologie du code. Le texte insiste ainsi sur l'apport des recherches « à grande échelle et décentralisés ", conduites collaborativement sur le modèle de Wikipédia. Il appelle en outre au développement de pratiques « itératives », fondées sur le « remix » et le « remontage perpétuel » (Schnapp et al., 2008).

Dans une veine similaire, inspirée par les textes de Marx sur le general intellect, David Berry (2015) insiste sur "la promesse d'un intellect collectif», rendu possible par les logiciels et le code. À travers ces citations, on voit que ce qui est valorisé est aussi bien la dimension collective de la production de connaissances, que le fait que cette production ne débouche pas sur des œuvres achevées, closes sur elles-mêmes, mais relève au contraire de processus ininterrompus d'enrichissement du savoir. Il s'agit là ni plus ni moins de la méthodologie dont les grandes communautés du logiciel libre (Linux, Debian, Apache, etc.), puis Wikipédia, ont montré depuis vingt ans la remarquable efficacité, afin de produire des « communs numériques " grâce aux contributions d'un grand nombre de volontaires (Broca, 2013). Le modèle promu est ainsi celui d'une science "wikifiée » ou "githubisée ${ }^{2}$ " (Maurel, 2015), conçue comme un work in progress permanent. Les exemples des logiciels libres et de Wikipédia, qui ont donné lieu à une importante littérature scientifique analysant leurs spécificités et les conditions de leur succès (Himanen, 2001 ; Weber, 2004 ; Benkler, 2006 ; Cardon, Levrel, 2009), sont assurément tout à fait remarquables. La fascination totalement exempte de distance qu'ils exercent sur certains théoriciens des humanités numériques ne manque pourtant pas d'étonner. Alors même qu'un informaticien comme Richard Stallman reconnaît qu'il existe différents types d'œuvres et plusieurs régimes de connaissance, qu'on ne peut donc simplement 
transposer le modèle du logiciel libre à toutes les productions de l'esprit (Stallman, 2002 : 141-163), certains partisans des digital humanities semblent considérer que les sciences humaines ne sauraient dorénavant exister que sur un modèle open source, par débogage progressif. C'est pourtant passer bien rapidement sur ce qui distingue la programmation d'un logiciel (grâce à une suite d'ajouts et de corrections) de l'échange d'arguments dans le cadre d'une discussion rationnelle ; ou bien considérer que cette dernière activité n'a plus lieu d'être. En d'autres termes, c'est importer dans le champ des sciences humaines un modèle purement cumulatif de construction du savoir, remplacer un idéal de confrontation raisonnée des points de vue par un modèle d'agrégation de savoirs locaux. On y perd quelque peu ses humanités, qu'elles soient numériques ou non.

Cette promotion d'une science collaborative et itérative est étroitement corrélée à une valorisation du «faire ». Le "manifeste pour des humanités numériques 2.0 » est très explicite sur ce point également. Après avoir critiqué «la dichotomie entre le royaume manuel de la fabrication et le royaume mental de la pensée », il vante «la fabrication physique, le design numérique, la stylisation élégante, la prose efficace, la juxtaposition des images, le montage du mouvement, l'orchestration des sons ", bref tout ce qui relève « du faire » (Schnapp et al., 2008). Ce renversement de la priorité accordée à la théorie sur la pratique est considéré comme l'un des principaux traits distinctifs des digital humanities . Comme l'affirme sans ambages Stephen Ramsay dans un texte en ligne, «si vous ne fabriquez rien, vous n'êtes pas [...] un humaniste numérique » (2011). L'insistance sur le «faire» renvoie en premier lieu, bien que non exclusivement, à l'informatique. «Fabriquer » des choses c'est d'abord faire du code et privilégier le hack au yack (Granjon, Magis, 2015 ; Granjon 2015). En outre, au-delà même des pratiques effectives auxquelles elle donne lieu, cette valorisation du «faire » apparaît comme une importation dans le champ des humanités de l'esprit hacker, tel que le décrit l'anthropologue Christopher Kelty: "L'éthique hacker ne descend pas des cimes de la philosophie comme l'impératif catégorique - les hackers n'ont pas de Kant et ils n'en veulent pas non plus. Bien plutôt, comme l'a suggéré Manuel Delanda, la philosophie du logiciel libre est le fait même du logiciel libre, ses pratiques et ses choses» (2008: 181). On ne saurait mieux définir l'épistémologie résolument empirique des HN.

24 En plus de fonctionner comme modèle méthodologique et incarnation privilégiée du «faire », le code a un troisième rôle. Il représente, pour certains théoriciens des HN, la promesse d'une unification du champ des sciences humaines grâce au développement d'une nouvelle forme d'interdisciplinarité. David Berry soutient ainsi que les « différentes disciplines universitaires pourraient trouver dans le computationnel un "noyau dur" ontologique très similaire", ce qui signifie que les "sciences computationnelles pourraient jouer le rôle de fondation envers les autres sciences » (Berry, 2015 : 199). Les technologies numériques permettraient donc, en unifiant les méthodes des différentes disciplines, de subsumer la diversité des recherches en sciences humaines sous l'unité d'une même forme « computationnelle » de connaissance. Il s'agit là, à vrai dire, d'une vieille antienne. Castoriadis écrivait déjà dans les années 1970 à propos du morcellement disciplinaire : «On a pu penser pendant longtemps, et encore aujourd'hui, que le dépassement de la séparation devait se faire et ne pouvait se faire que par l'unification des méthodes fondamentales dans les différents domaines, ou par la réduction de ceux-ci à un domaine élémentaire» (1998: 262-263). Seule la définition de ce «domaine élémentaire » a changé : la physique mathématique a autrefois pu prétendre tenir ce rôle, il s'agit désormais des sciences informatiques. Le projet n'en reste pas moins aussi peu 
convaincant aujourd'hui qu'hier. Certes, rien n'empêche de traiter tous les types de problèmes et de questions grâce à des méthodes algorithmiques. Il est même probable que cela produise parfois des résultats intéressants et des rapprochements inédits entre disciplines. On se tromperait toutefois en pensant ne rien y perdre, particulièrement au sein des sciences humaines, lorsqu'il s'agit d'élucider cet objet spécifique qu'est le socialhistorique.

\section{Apports et limites de la critique par le hacking}

Il apparaît donc que la rationalité algorithmique et l'imaginaire de l'informatique infusent profondément les $\mathrm{HN}$, en déterminant dans une mesure non négligeable les méthodes et le rapport au savoir que celles-ci revendiquent. Une dernière question demeure : quel rapport cette prégnance de l'épistémologie du code entretient-elle avec la question de la critique en sciences humaines? Une précision liminaire s'impose ici. On aurait tort de croire que l'épistémologie du code s'oppose purement et simplement à la critique, ou de prendre tous les informaticiens pour des serviteurs dociles de la «société de l'information ». L'importance politique et culturelle du mouvement du logiciel libre suffit à suggérer que de tels jugements sont pour le moins hâtifs (Kelty, 2008; Broca, 2013). On remarquera en outre que la posture des "humanistes numériques" relativement à la critique est loin d'être claire et uniforme. Comme le relève Richard Grusin dans un texte en ligne, ceux-ci font parfois de la valorisation du « faire » une arme pour « discréditer la critique (ou par extension toute autre forme de recherche humaniste qui ne fabrique rien) » (2013). Mais on rencontre aussi des tenants des digital humanities, qui entendent coupler compétences en programmation et distance critique, voir développer une forme de "critique algorithmique» (Ramsay, 2008). David Berry fait assurément partie de cette deuxième catégorie. Il propose ainsi de développer une "pratique critique articulée à la possibilité concrète de hacker, pirater, bidouiller, bloquer et reconfigurer de nouveaux cheminements et de nouveaux possibles» (2015: 203). Autrement dit, il s'agit pour lui de mettre en question le monde logiciarisé qui est le nôtre à travers la subversion de ses "systèmes computationnels ", en cessant - de manière tout à fait concrète - de considérer les logiciels comme des boîtes noires. Berry se place donc sur un terrain qui est autant épistémologique que politique. Il présente le hacking comme une manière de comprendre les arcanes des systèmes informationnels qui nous entourent, mais aussi comme un moyen de redonner aux individus du pouvoir sur leur environnement.

Cette proposition théorico-pratique, pour intéressante qu'on puisse la trouver, n'est guère originale, lorsqu'on connaît un minimum la culture hacker et l'histoire du mouvement du logiciel libre. Elle correspond peu ou prou à ce que répètent les partisans du free software - et notamment le premier d'entre eux, Richard Stallman - depuis le début des années 1980. La culture hacker repose en effet sur l'idée que la véritable apprentissage n'est pas abstrait, mais se donne comme un " apprentissage par l'objet » (Auray, 2000: 84), c'est-à-dire par la pratique, la «bidouille » et les tâtonnements. De plus, les "libristes" insistent depuis longtemps sur la manière dont l'inculture informatique du grand public est la conséquence des pratiques "propriétaires" de l'industrie du logiciel, et sur les vertus du hacking pour redonner aux individus davantage de libertés et de maîtrise sur leur environnement (Stallman, 1986). Les tenants 
d'humanités numériques critiques apparaissent donc ici autant à la remorque des informaticiens contestataires qu'à l'avant-garde des humanistes engagés.

Mais là n'est pas le véritable problème. Ce qui interroge, ce sont les limites structurelles d'une critique de la logiciarisation du monde qui veut se faire avec les outils même de ce qu'elle entend intellectuellement mettre à distance. Pour le dire simplement: jusqu'à quel point peut-on tenir un discours critique sur l'informatisation grâce à l'informatique? La réponse à cette question peut se penser à travers les notions de critique interne et de critique externe. Quand la première est adossée aux valeurs du champ social qu'elle prend pour objet, la seconde s'appuie sur des principes ou des positions normatives étrangères à celui-ci (Boltanski, Chiapello, 1999 : 76-77). La critique portée par les humanités numériques est définitivement du premier type. Elle ne remet jamais fondamentalement en cause la logique d'informatisation de nos sociétés. Son discours comme ses pratiques restent prisonnières des formes cognitives et des schèmes imaginaires liés à l'épistémologie du code. Elle opère donc dans une logique de réforme, éventuellement de subversion. Cela n'est assurément pas dénué d'intérêt. Cela demeure toutefois structurellement limité, dès lors que les outils intellectuels (concepts, méthodologies, etc.) dont il est fait usage sont imprégnés par la rationalité et l'imaginaire du champ social abordé. On est loin du projet que Castoriadis assignait aux intellectuels : celui d'une mise en question radicale de l'institué, c'est-à-dire des significations imaginaires sociales (Castoriadis, 2005 : 102). Le point le plus troublant dans le discours des $\mathrm{HN}$ est toutefois moins qu'il demeure en deçà de ce projet exigeant, que le fait qu'il veuille le disqualifier a priori. Tel est en effet aussi le sens de l'injonction à "coder ", manière de signifier que tout discours valable devrait désormais s'inscrire dans le cercle de la raison algorithmique, et que tout scientifique sérieux devrait dorénavant être instruit des méthodes computationnelles et, de préférence, en faire usage. On rappellera par conséquent ce qui semble pourtant tenir de l'évidence: le développement d'humanités critiques du monde numérique ne passe pas nécessairement par le développement d'humanités numériques.

L'épistémologie du code se présente donc comme la conjonction de la rationalité algorithmique des ordinateurs et de l'imaginaire de l'informatique. Dans sa forme pure, elle consiste à penser que tous les phénomènes du monde naturel et du monde social sont redevables d'une approche computationnelle. Les humanités numériques en apparaissent imprégnées, dès lors qu'elles empruntent dans une large mesure leur conception du savoir, leurs concepts et leurs méthodes au champ informatique et, plus particulièrement me semble-t-il, à la culture hacker et aux projets open source. L'irruption de cette épistémologie dans le champ des sciences sociales pose question, dans la mesure où elle conduit à occulter la spécificité ontologique du social-historique, en tant que monde des significations imaginaires. Contre cette forme de réductionnisme, il faut donc rappeler que les techniques de recherche informatiques ne sauraient être des «baromètres du social» (Gillespie, 2014). Elles reposent nécessairement en amont sur une opération de "purification » de la signification, et requièrent en aval des formes d'interprétation qui ne peuvent être totalement déléguées aux machines. Dire cela ne revient ni à jeter l'opprobre sur le travail des informaticiens, ni à nier l'apport qui peut être le leur dans le champ des sciences sociales. Il s'agit plutôt de défendre une forme de vigilance 
épistémologie, face à la tentation de voir dans le code l'alpha et l'omega de toute démarche scientifique valide. Comme le souligne Katherine Hayles, la formalisation informatique permet souvent «de mettre au clair certaines implications, de les examiner et de les modifier d'une manière qui ne serait peut-être pas possible avec l'écrit ", mais elle est en revanche incapable de saisir «le bruit d'un monde trop plein d'ambiguïtés et de complexité » (2012: 42). En effet, si l'idéal de transparence et d'univocité est atteignable dans le monde du code, il est fondamentalement étranger au monde social, hormis comme mythe ou schème imaginaire.

Afin de résister aux tentations impérialistes de l'épistémologie du code, il convient par conséquent de défendre la légitimité de "stratégies rationnelles non computationnelles " (Bachimont, 2004), ce par quoi l'on entendra essentiellement deux choses: l'argumentation et la narration. L'argumentation est ce qui empêche de réduire le travail des sciences humaines à un gigantesque problem solving, en reconnaissant que dès lors que ces disciplines ont pour enjeu "la représentation légitime du monde social » (Bourdieu, 1976), elles doivent laisser une place à la confrontation raisonnée des points de vue. La narration est ce «qui éveille et affine la capacité à voir le monde à travers les yeux d'autrui » (Nussbaum, 2010:121) et permet par conséquent de dépasser quelque peu l'éclatement des vécus individuels, en ouvrant un accès à la multiplicité des expériences singulières. Ce n'est, me semble-t-il, qu'en cultivant ces diverses stratégies, que les humanités pourront continuer à dire, sans jamais l'épuiser, la richesse du monde social.

\section{BIBLIOGRAPHIE}

Andreessen (Marc), « Why Software Is Eating The World », Wall Street Journal, 20 août 2011, http://www.wsj.com/articles/SB10001424053111903480904576512250915629460.

Auray (Nicolas), Politique de l'informatique et de l'information. Les pionniers de la nouvelle frontière électronique, thèse de sociologie dirigée par Luc Boltanski, École des Hautes Études en Sciences Sociales, 2000.

Bachimont (Bruno), «Signes formels et computation numérique : entre intuition et formalisme. Critique de la raison computationnelle », 2004, http://www.utc.fr/ bachimon/

Publications_attachments/Bachimont.pdf.

Benkler (Yochai), The Wealth of Networks. How Social Production Transforms Markets and Freedom, New Haven, Yale University Press, 2006,

Boltanski (Luc) \& Chiapello (Eve), Le nouvel esprit du capitalisme, Paris, Gallimard, 1999.

Berry (David M.), « Subjectivités computationnelles », Multitudes, n 59, été 2015, 2015, pp. 196-206.

Bourdieu (Pierre), «Le champ scientifique », Actes de la recherche en sciences sociales, n²-3, 1976, pp. 88-104.

Breton (Philippe), Une histoire de l'informatique, Paris, Seuil, 1990. 
Broca (Sébastien), Utopie du logiciel libre. Du bricolage informatique à la réinvention sociale, Neuvy-enChampagne, Le passager clandestin, 2013.

Cantor (Georg), Beiträge zur Begründung der transfiniten Mengenlehre, Leipzig, Teubner, 1895.

Cardon (Dominique), Levrel (Julien), « La vigilance participative. Une interprétation de la gouvernance de Wikipédia », Réseaux, vol. 2, n 154, 2009, pp. 51-89/

Castoriadis (Cornelius), L'institution imaginaire de la société, Paris, Seuil, 1975.

Castoriadis (Cornelius), Les carrefours du labyrinthe 1, Paris, Seuil, 1998 (1978).

Castoriadis (Cornelius), Domaines de l'homme (Les carrefours du labyrinthe 2), Paris, Seuil, 1986.

Castoriadis (Cornelius), Le monde morcelé (Les carrefours du labyrinthe 3), Paris, Seuil, 1990.

Castoriadis (Cornelius), La montée de l'insignifiance (Les carrefours du labyrinthe 4), Paris, Seuil, 1996.

Castoriadis (Cornelius), Fait et à faire (Les carrefours du labyrinthe 5), Paris, Seuil, 1997.

Castoriadis (Cornelius), Une société à la dérive, Paris, Seuil, 2005.

Castoriadis (Cornelius), L'imaginaire comme tel, Paris, Hermann, 2007.

Coleman (Georg), « The Political Agnosticism of Free and Open Source Software and the Inadvertent Politics of Contrast ", Anthropological Quarterly, vol. 77, n³, été 2004, pp. 507- 519.

Fuller (Matthew), Software Studies. A Lexicon, Cambridge, MIT Press, 2008.

Galloway (Alexander), Protocol : how control exists after decentralization, Cambridge, MIT Press, 2004.

Gillespie (Tarleton), « The Relevance of Algorithms », in Gillespie (Tarleton), Boczkowski (Pablo J.), Foot (Kirsten A.), A., Media Technologies. Essays on Communication, Materiality and Society, Cambridge, MIT Press, 2014, pp. 167-195.

Goody (Jack), La raison graphique : la domestication de la pensée sauvage, Paris, Éditions de Minuit, 1978.

Granjon (Fabien), « Du pragmatisme et des technologies numériques », Hermès, n 73, 2015, pp. 215-220.

Granjon (Fabien), Magis (Christophe), « Vers une "nouvelle anthropologie” critique. Jalons pour une épistémologie matérialiste des humanités numériques ", Journal des Anthropologues, $\mathrm{n}^{\circ}$ 142-143, 2015, pp. 281-303

Grusin (Richard), « The Dark Side of the Digital Humanities - Part 2 », 9 janvier 2013, http:// www.c21uwm.com/2013/01/09/dark-side-of-the-digital-humanities-part-2/

Guichard (Éric), «L'internet et les épistémologies des sciences humaines et sociales », Sciences/ Lettres, $\mathrm{n}^{\circ} 2,2014$, http://rsl.revues.org/389

Hayles (Katherine), My Mother was a Computer. Digital Subjects and Literary Texts, Chicago, University of Chicago Press, 2005.

Hayles (Katherine), How We Think. Digital Media and Contemporary Technogenesis, Chicago, University of Chicago Press, 2012.

Himanen (Pekka), L'éthique hacker, Paris, Exils, 2001.

Hobbes (Thomas), Léviathan, Paris, Gallimard, 2000.

Kelty (Christopher M.), Two Bits. The Cultural Significance of Free Software, Durham, Duke University Press, 2008. 
Manovich (Lev), Software Takes Command, New York, Bloomsbury, 2013.

Maurel (Lionel), « La wikification de la science comme nouvel horizon pour l'Open Access », 12 août 2015, http://scinfolex.com/2015/08/12/la-wikification-de-la-science-comme-nouvelhorizon-pour-lopen-access/

Nussbaum (Martha C.), Les émotions démocratiques. Comment former le citoyen du XXIe siècle, Paris, Flammarion, 2011.

Ramsay (Stephen), «Algorithmic Criticism », in Schreibman (Susan), Siemens (Ray) eds., A Companion to Digital Litterary Studies, Oxford, Blackwell, 2008.

Ramsay (Stephen), « Who's In and Who's Out », 8 janvier 2011, http://stephenramsay.us/ text/2011/01/08/whos-in-and-whos-out/.

Rouvroy (Antoinette), « Pour une défense de l'éprouvante inopérationnalité du droit face à l'opérationnalité sans épreuve du comportementalisme numérique », Dissensus, $\mathrm{n}^{\circ} 4$, avril 2011, http://popups.ulg.ac.be/2031-4981/index.php?id=963.

Rouvroy (Antoinette), Stiegler (Bernard), « Le régime de vérité numérique. De la gouvernementalité algorithmique à un nouvel État de droit », Socio, n 4, 2015, pp. 113-140.

Schnapp (Jeffrey) et al., « The Digital Humanities Manifesto 2.0 », http:// www.humanitiesblast.com/manifesto/Manifesto_V2.pdf, 2008 ; repris et traduit dans Multitudes, n 59, été 2015, pp. 181-196.

Stallman (Richard M.), « Lecture at Kungliga Tekniska Högskolan (KTH) », Stockholm, 30 octobre 1986, http://www.gnu.org/philosophy/stallman-kth.html

Stallman (Richard M.), Free Software, Free Society : Selected Essays of Richard M. Stallman, Boston, GNU Press, 2002.

Tomès (Arnaud), « Commentaire » in Castoriadis C., L'imaginaire comme tel, Paris, Hermann, 2007. Varela (Francesco J.), Connaître les sciences cognitives. Tendances et perspectives, Paris, Seuil, 1988.

Waquet (Françoise), L'ordre matériel du savoir, Paris, CNRS Éditions, 2015.

Weber (Steven), The Success of Open Source, Cambridge : Harvard University Press, 2004.

Wing (Jeannette M.), «Computational Thinking », Communications of the ACM, vol. 49, $\mathrm{n}^{\circ}$ 3, 2006, https://www.cs.cmu.edu/ 15110-s13/Wing06-ct.pdf .

Wolfram (Stephen), A New Kind of Science, Champaign, Wolfram Media, 2002.

Zimmermann (Jérémie), intervention à la séance de conférences « Logiciel libre et économie de la contribution : le temps de la déprolétarisation » organisée par l'association Ars Industrialis , Paris, 6 mars 2010 .

\section{NOTES DE FIN}

1. http://www.gnu.org/philosophy/words-to-avoid.html

2. GitHub est « un service web d'hébergement et de gestion de développement de logiciels, utilisant le logiciel de gestion de versions Git » (Wikipédia, https://fr.wikipedia.org/wiki/GitHub ) . 
INDEX

Mots-clés : algorithme, code, hacker, imaginaire, institution sociale

\section{AUTEUR}

\section{SÉBASTIEN BROCA}

Maître de Conférences en Sciences de l'information et de la communication, Université Paris 8 Cemti 